\title{
Blockchain and Internet of Things for Business Process Management: Theory, Challenges, and Key Success Factors
}

\author{
Mabrook S. Al-Rakhami ${ }^{1}$, Majed Al-Mashari ${ }^{2}$ \\ Information Systems Department \\ College of Computer and Information Sciences \\ King Saud University, Riyadh, Saudi Arabia
}

\begin{abstract}
The combination of business process management (BPM) and emerging technologies is a logical step. The evolving number of advanced technologies shows that contributions are with a high value for business processes. From a BPM point of view, value creation from the modern technologies such as Internet of things and Blockchain technology is pivotal on a progressively higher scale and affect the business process in different aspects. However, current research in this area still at the beginning. In order to close this research gap and the lack of experience in this area, the topic of integrating Blockchain and IoT technologies with BPM will play an essential role in a corporate context, particularly in the context of inter/intraorganizational information systems and their diverse design options. This review paper aims to survey the impact of tow emerging technologies: Internet of Things and Blockchain technology on BPM and illustrates the current state of the art in this research domain. Each technology was investigated through a design science research approach to provide as a descriptive theoretical overview, characterize its theoretical background, challenges, and key success factors.
\end{abstract}

Keywords-Business process management; BPM; Internet of things; IoT; blockchain technology

\section{INTRODUCTION}

The development of business at the national and international levels requires processes that dominate market instruments. Imagination, creativity, and technical instruments should be at the highest level to meet the market needs and high international competition. Today, selling alone is not enough. The client is given what he wants and needs at the right moment. Hence, thinking strategically is also necessary for relational marketing with advertising support to obtain a perfect mix in the competitiveness of companies [1].

In these times of global and ongoing progression of digital transformation, the evaluation of emerging technologies and the study of their potential research and application domains are of significance. Organizations have to develop their core process to remain competitive, transform their businesses, and stay more effective. Sometimes, they even need to change the entire business models.

By reviewing relevant publications [1-12], this study develops a clear articulation of the concept, nature, components, and elements of business process management (BPM), which is significant in understanding the BPM research better. With the rapid growth of the volume of research output on the BPM topic [7], this study considers two recent technologies to draw conclusions on the current status and possible future directions.

Over the last decade, many technologies have emerged as promising paradigms for integrating and extending business processes at both intra- and inter-organizational levels, which are well known as industry 4.0. These emerging technologies have been fueled by the recent development of information and communications technology. The developments and technological advances will provide a viable array of solutions to the growing needs of information in different units of organizations. This viability has been evidenced by the fact that a growing number of enterprises worldwide have explored the benefits of digitizing enterprises' horizontal and vertical chains and adopted recent and advanced technologies in the process of becoming leading digital enterprises in tomorrow's complex industrial ecosystems.

Various technologies can be used for implementing the fourth industrial revolution (Industry 4.0) which affect strongly how BPM works. These technologies include the Internet-ofThings (IoT), blockchain, embedded systems, wireless sensor network, industrial robots and artificial intelligence among other technologies. In this article, two selected technologies are selected which are particularly significant for BPM. These technologies are the IoT and Blockchain technology. However, the coverage of all enabling technologies is by no means meant to be exhaustive. In this regard, this review article is designed to answer the following questions, which are as follows: How the selected technologies affect the IT aspect of BPM?, and what are the challenges for the application of these technologies? Moreover, what are the key factors to ensure the successful application of these technologies for BPM?

The contribution of this article is threefold: First, this work presents a survey on how BPM could be integrated with two of the recent technologies which are IoT and Blockchain. Second, it highlights the challenges that face the integration process. Third, it describes and discuss the key success factors of the integration between the technologies under investigation.

The remainder of this paper is structured as follows: Section II describes research methodology. Section III provides a theoretical background of the BPM by considering the BPM 
six-core element model. Section IV elaborates on the fundamental concepts of the selected technologies, namely IoT and Blockchain technology, where each sub-section presents the overview, challenges, and key success factors for the abovementioned technologies. Section $\mathrm{V}$ provides a discussion on final thoughts and key point summary. Finally, section VI concludes the paper and presents the future work.

\section{MATERIALS AND METHODS}

The objective of this article is to investigate the impact of two emerging technologies on BPM, precisely, to study their challenges and key success factors. In order to achieve this objective, and to formulate the scope for the reviewed papers, this work adapted the steps shown in Fig. 1.

As a first step, the search for selected keywords related to recent technologies associated with BPM has been conducted on the indexed Web of Sciences (WoS) dataset. WoS is a recognized multidisciplinary proprietary database for peerreviewed journal content. In addition, it has a powerful feature of tracking citing items. WoS is mainly used because it searches over 33,000 journals and over 21,000 books and conference proceedings, which was sufficient for this study.

The next step was to analyze the highly cited articles and investigate the mentioned challenges and key success factors for the application of selected topics with BPM direction. Although challenges and key success factors weren't necessarily mentioned directly, so it was necessary to figure it out based on our understanding of the discussions and conclusion of these articles. Table 1 illustrates the used queries for each topic and the statistics of retrieved articles. The query was carried out from All Databases.

For each topic, the number of related publications, average citations per item, the sum of times cited and the number of citing articles are calculated as shown in Table 1. Figs. 2 and 3 show the number of publications per topic. Figures demonstrate the importance of the selected technologies over the years, where the increase in the number of publications over the years is clear.

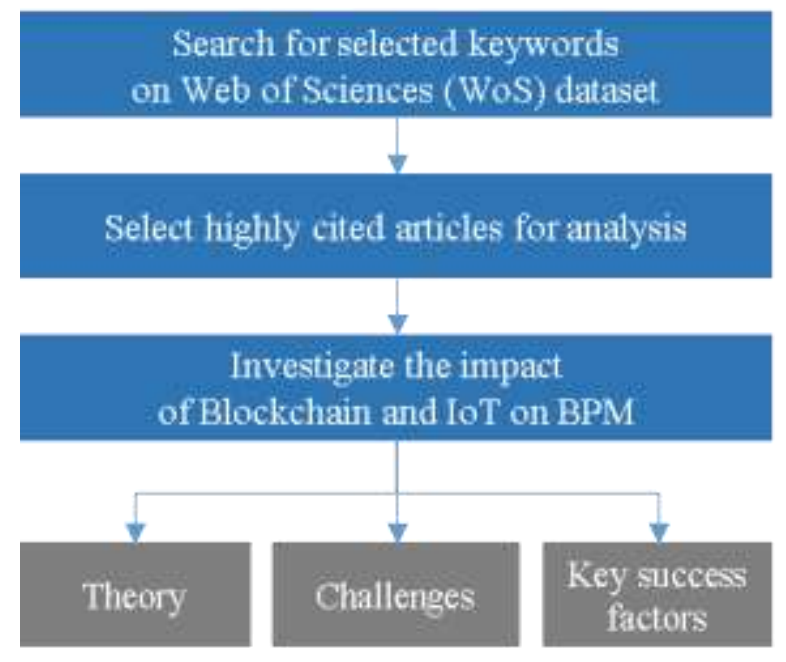

Fig. 1. Research Methodology.

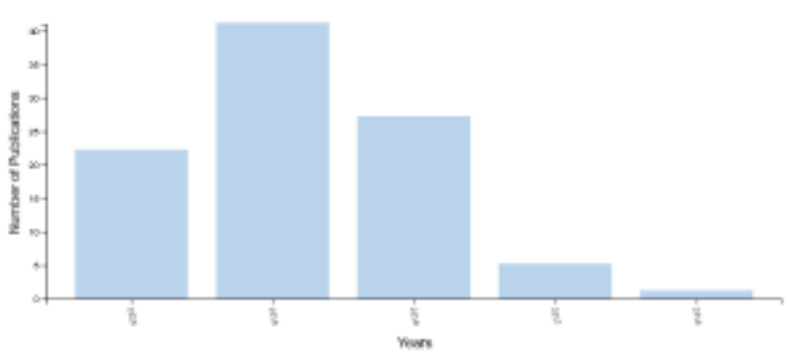

Fig. 2. Blockchain Technology for Business Process Management Publications Count per Year.

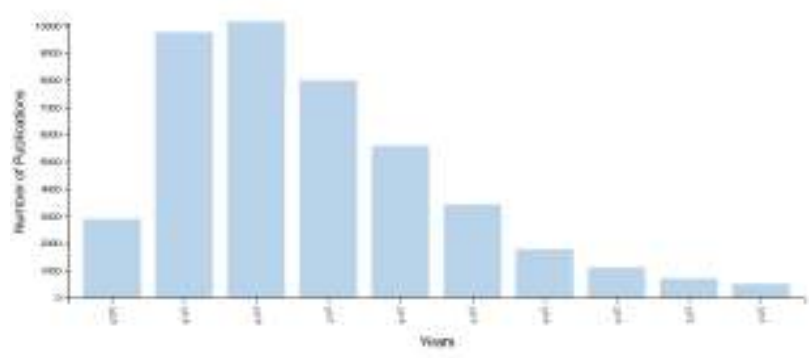

Fig. 3. IoT-based Business Process Management Publications Count per Year.

TABLE I. WOS QUERY KeYwordS AND STATISTICS OF THE SELECTED TECHNOLOGIES RELATED TO BPM

\begin{tabular}{|c|c|c|c|c|}
\hline Topic & $\begin{array}{l}\text { Total } \\
\text { publications }\end{array}$ & $\begin{array}{l}\text { Average } \\
\text { citations } \\
\text { per item }\end{array}$ & $\begin{array}{l}\text { Sum of } \\
\text { times } \\
\text { cited }\end{array}$ & $\begin{array}{l}\text { Citing } \\
\text { articles }\end{array}$ \\
\hline $\begin{array}{l}\text { TOPIC: ('business process } \\
\text { management' OR 'BPM') } \\
\text { AND inTITLE: } \\
\text { ('Blockchain') }\end{array}$ & 176 & 1.75 & 308 & 244 \\
\hline $\begin{array}{l}\text { TOPIC: ('business process } \\
\text { management' OR 'BPM') } \\
\text { AND inTITLE: (IoT OR } \\
\text { 'Internet-of-Things' OR } \\
\text { 'Internet of Things') }\end{array}$ & 298 & 4.05 & 1,208 & 1,105 \\
\hline
\end{tabular}

\section{BPM: THE ORETICAL BACKGROUND}

\section{A. Motivation and Definition}

The competitiveness of companies depends on improving their efficiency, increasing productivity, reducing costs, matching products/services to customer expectations, the global vision between different processes, and the strategic alignment of their information system [14]. This requires an organization of the company around strategic and transversal processes.

BPM has recently received considerable attention from both business administration and computer science communities [10]. BPM is a discipline that combines methods, technologies, and tools to handle the entire life cycle of business processes in a single approach, collaborating systems, software, and people. BPM can be defined as "supporting business processes using methods, techniques and software to design, enact, control and analyze operational processes involving humans, organizations, applications, documents and other sources of information" [15]. 


\section{B. BPM Six-Core Elements Model}

Several theorists wrote about the key elements of business processes. They generally agree on identifying six basic elements that must be evaluated for business process management: strategic alignment, government, methods, information technologies, people, and culture. Fig. 4 illustrates a further level of detail for the six core elements associated with their capability areas.

1) Strategic alignment: Strategic alignment is defined as the close link of the priorities of the organization and processes of the company that allows a continuous and effective action to improve the business performance. Five different capacity areas are identified as part of an assessment of the strategic alignment of business processes.

2) Government: The government of BPM is dedicated to accountability in an orderly and transparent manner in terms of the roles and responsibilities of the different BPM levels (portfolio of programs, projects, and operations).

3) Methods: In the context of BPM, methods are defined as the tools and techniques that support and allow consistent activities at all BPM levels (portfolio of programs, projects, and operations). Different methods can be performed in the most important stages in the different stages of their life cycle.

4) Information technology: Information technology (IT) refers to software, hardware, and information systems that enable processes. The decomposition of IT as one of the basic elements of BPM is similar to that of BPM methods and also refers to the stages of the life cycle process. Like the dimension of methods, IT components focus on the specific needs of each stage of the process cycle and are evaluated from certain points of view.

5) People: People are defined as individuals and groups that continuously improve and use their expertise, ability to manage processes, and knowledge to improve business performance.

6) Culture: Culture refers to the collective values and beliefs that shape the processes related to attitudes and behavior to improve business performance.
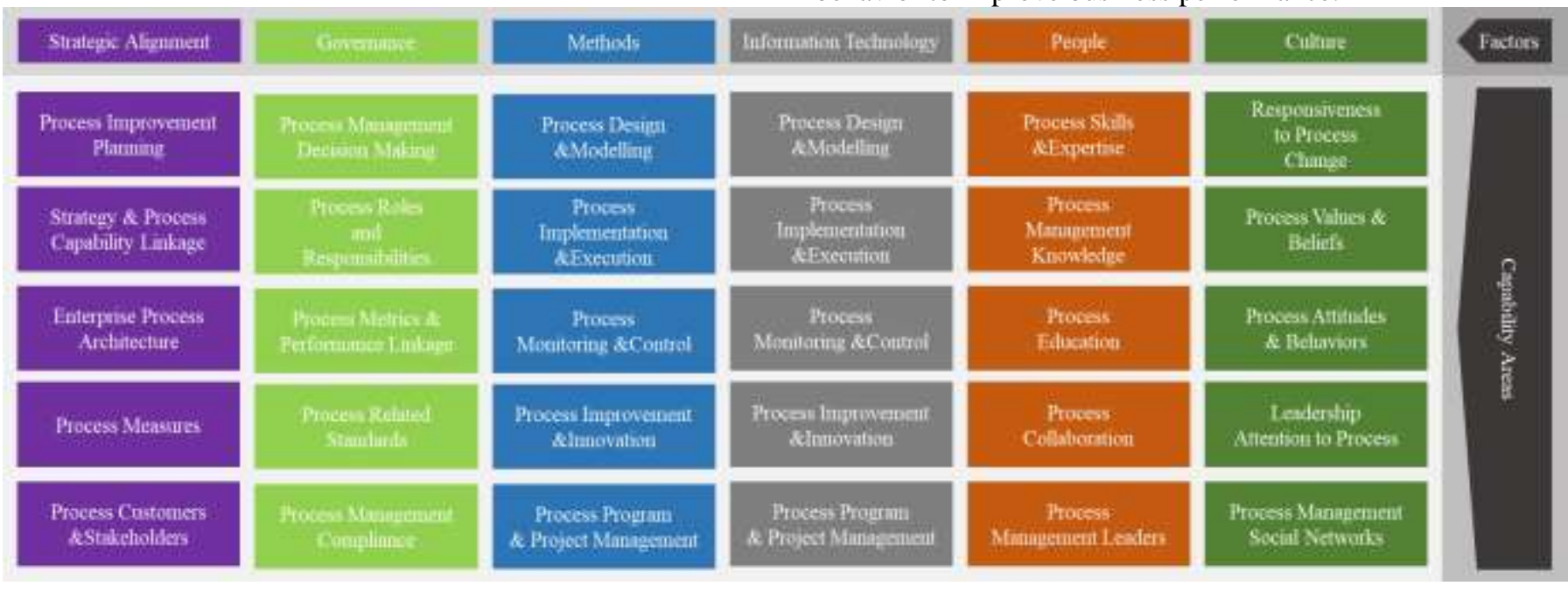

Fig. 4. Six Core Elements of BPM. Adapted from [2].

\section{EMERGING TECHNOLOGIES}

BPM continues to advance throughout the world and evolves because of the great advancement of new technologies, such as IoT, cloud computing, Big Data, social BPM, etc. the growing importance is given to the management of processes. However, it is not only about technologies, but also about managing business processes and resources, which should be aligned with the business strategy and achieving a true and complete business process management [16]. These same technologies are also achieving digital transformation, which cannot be separated from business processes, because everything is related to processes. These must be managed and aligned with the business strategy [17].

This section introduces an overview of the two technologies that have a focus of the emergent body of research in BPM: IoT and Blockchain technology. In accordance with the advances that continuously occur in both information systems and global business strategies, these technologies have emerged to improve the productivity and quality of business and its process in organizations of different sizes and operations. Fig. 5 presents the structure of this work.

\section{A. Internet of Things}

1) Theoretical overview: The vision of IoT will become the center of privacy and business in the future because billions of networked objects can communicate with each other [18]. The use of state-of-the-art technologies enables the emergence of new applications. Having instant access to information on the physical world and objects increases efficiency and productivity, creating great opportunities for business and personal life. The resulting new requirements also require existing business processes to be adapted to realize the full potential of IoT [19]. 


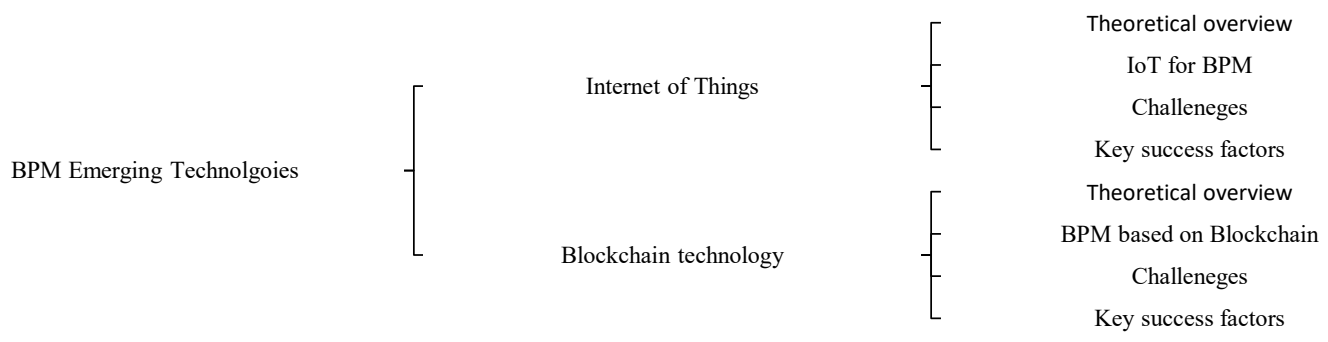

Fig. 5. Paper Structure.

This section deals with the concept of IoT and creates a holistic understanding of the topic. Therefore, both technologies and application scenarios, as well as the processes related to this topic, are presented and explained. The goal is to derive requirements for process support and technology compared to other works in the field and illustrate the impact of the IoT on processes. The use of IoT makes it possible to consider extensive real-time information in business processes. The result is more transparent processes and monitoring of business processes [20].

IoT is still at the beginning of its development. Every day, more machines, containers, houses, vehicles, and people are equipped with sensors to report their status, receive instructions, or even take action based on the information they receive. IoT has the potential to fundamentally change the way people interact with their environment. The ability to electronically monitor and control objects in the physical world enables automated, data-driven decision-making to optimize system and process performance, increase enterprise efficiency, and improve the quality of life. From monitoring machinery in production halls to tracking products on the high seas, sensors can help companies make better, more efficient use of their assets [21]. IoT also has the potential to significantly change or improve processes and procedures in private households or in the healthcare industry, especially in the treatment of chronically ill patients, such as those with iabetes. IoT can dramatically change the reach of information technology by blurring the physical real world with the digital world. To achieve this, technical and organizational hurdles must be overcome, and all the existing business processes have to be changed. In particular, companies that want to use the technologies of IoT in the future need better tools and methods to analyze the existing processes and adapt them to IoT [9].

In an environment of constant competition and ever-faster innovation, many companies are in a permanent process of change. Even in a company with a degree of stability, operational and technical processes need to be continuously improved and adjusted. Therefore, processes in a company have a high priority. However, the processes have to be adapted to the new possibilities of IoT to realize their full potential. The right people must use the right objects in the right situation at the right time. Hence, all parties involved, particularly people, systems, machines, and smart objects, must be coordinated. Countless sensors on devices in the industry, in cities, homes, and cars create a huge amount of real-time data that needs to be analyzed. Networks can exchange these data and make decisions based on the processing logic. However, this logic only works according to previously planned rules of conduct. Unforeseen exceptions based on real events require a dynamic process adaptation.

Therefore, the use of IoT requires flexible business processes that are capable of dynamically adapting to the changes that can occur through process execution or the properties of the smart objects [22]. While some call IoT a short-term hype, there is no question about its spread. Gartner estimated that today, more than 8 billion devices, including smartphones and computers, are connected around the world. This number is expected to dramatically increase to as much as 25 billion over the next ten years. IoT attempts to coordinate this enormous number of different devices with people and applications through business processes. The goal is to close the gap that exists between business processes in the real world and the information systems in the digital world. Fig. 6 represents the BPMS framework that encompasses IoT entities and the middleware-based information system. Most IoT-based BPM frameworks are similar to the design in this figure [1].

2) Challenges: Processes: The deployments of IoT technologies would considerably affect and modify how businesses do business amid diverse segments of the social order, affecting several processes [13]. For being capable of gaining numerous latent profits that are proposed for the IoT, numerous challenges concerning the modeling and implementation of these kinds of processes must be resolved to comprehend the broader and profitable deployments of IoT $[20,23]$. The exceptional features of IoT services and processes must be considered. In addition, current business process modeling and execution and service description languages such as Unified Service Description Language (USDL) will probably be required to be protracted [24].

Adaptive and Event-driven Processes: Note that the processes get more accustomed to what is taking place in the actual world-chief benefits of IoT integration. Characteristically, it is founded on happenings identified either unswervingly or through a real-time study of sensor data. These kinds of events can occur in the process at any time [25, 26]. The probability of occurrence for certain events is extremely low, indicating their possibility to take place, but not when or if they will take place [27]. 


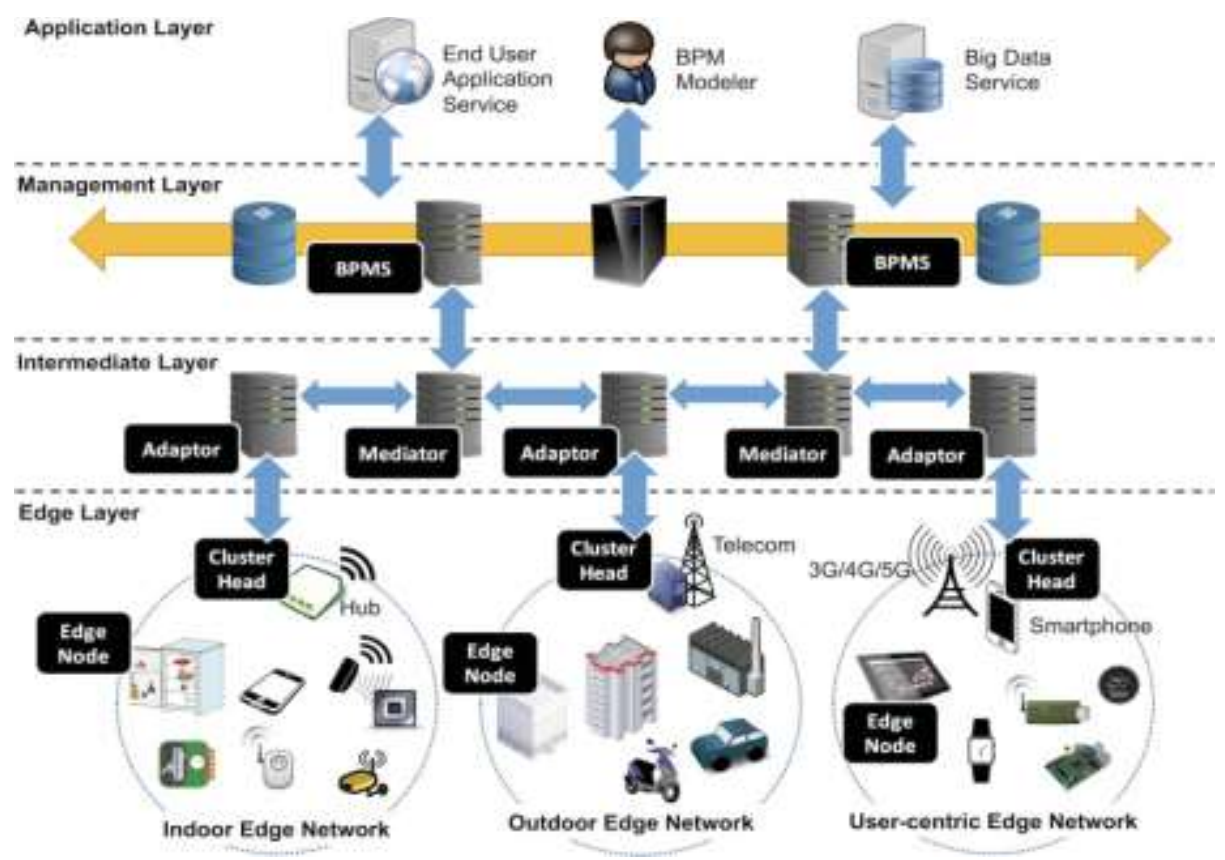

Fig. 6. BPM for the IoT Framework, Adapted from [1].

Modeling these kinds of events in a process is burdensome because these events must be encompassed in all probable activities, resulting in added difficulty as well as making it hard to comprehend the modeled process, specifically the chief process flow. Furthermore, the context will be considered to decide on how to respond to a lone event, the events that have been formerly perceived [28]. The study on event-driven and adaptive processes in IoT systems can reflect the extension and use of event-driven architectures (EDA) for activity checking and complex event processing (CEP). To start particular parts or steps of a business process, EDA can be joined with business process execution languages [29].

Processes Dealing with Unreliable Data: While working with events that are approaching from the physical world (like from signal-processing algorithms or sensors), some amount of undependability and doubt is put in the processes. In case the events with a certain doubt in them are used as a basis to take the conclusions in a business process, it is sensible to link all these events with a certain value for Quality of Information (QoI) [30].

In easy cases, this lets the process modeler describe thresholds. For instance, the event is presumed to have actually taken place if the degree of certainty is above $95 \%$. In case it is amid $60-95 \%$, we start to find out if certain other activities took place or not. The event is disregarded in case it is under $60 \%$. Things become more difficult in cases when numerous events are involved. For instance, the degree of certainty of one event is 65\%; one has $98 \%$; and another has $83 \%$. The principal services that fire the actual events need to be programmed to link these kinds of QoI values with the events [31]. From the viewpoint of a BPM, this kind of information must be taken, treated as well as articulated in a language like BPMN, which is a modeling notation language. Furthermore, the semantics and syntax of these kinds of QoI values must be standardized. As for the examples given earlier, is it merely a certainty percentage, or must it be more expressive (like a range in which true value is there)? Applicable methods must not only merely look in the uncertainty flow of a business process based on IoT, but also in the total modeling and structuring of (perhaps unstructured or unknown) process flows. Practices for uncertain modeling of processes and data can be considered [32,33].

Processes Dealing with Unreliable Resources: The data from resources is characteristically undependable. The resources offering the data are also undependable because of the hosting device's failure [34]. Processes depending on these kinds of resources are required to be capable of adapting to these kinds of conditions. The major subject is to identify this kind of a failure. The detection, in which a process is calling a resource openly, is unimportant. It is tougher in the case of resources that may produce an event at one point in time (for instance, the resource that observes the temperature in the truck gives an alert in case it gets extremely hot) [35]. Resource failure can be a reason for not getting any event; however, one other reason can be that nothing was there to report. Similarly, the generated reports' quality must be frequently examined for accuracy. Certain monitoring software is required to sense these kinds of problems; however, if this kind of software must be a separate component or a segment of the BPM execution environment is unclear. Amid the challenges of the research is the management of monitoring processes with run-time actuating processes, provided that management planes have a tendency to function at diverse time scales from IoT processes [33].

Highly Distributed Processes: Performing a process in a decentralized manner is important; at the time, the interaction with real-world devices and objects is needed. The decentralization and the decomposition of current business processes upsurge performance and scalability and result in better decision making. It can even result in novel business 
models. For instance, no messages are required to be sent to the central system in supply chain tracking or environmental monitoring applications if everything is in the limits. In case of a deviance, an alert (event) that can result in the adaptation of the whole process must be produced [34].

From the viewpoint of business process modeling, the process can probably explained centrally, providing that certain activities would be remotely performed. When the whole process is exhibited, linked services can probably be installed, which are to be implemented after this run and monitor the whole process. Appropriate issues of research comprise techniques and tools for the synthesis, authentication, and alteration of distributed processes in a volatile environment (e.g., altering settings, movement, and devices/objects connected to internet that leave or join) [36].

3) Key success factors: Build IoT-BPM Ecosystems: To control all aspects of the challenges that the BPM based on IoT, business ecosystems that are comparable to a coral reef, where a diversity of species can be found living in symbiosis with a shared development, must be built. This ecosystem must be friendly to all those who integrate it based on collaboration. The ecosystem allows every organization to stay alive and adapt to changes. Decisions are made as a team, and competitors are often partners too, sharing market and values [36].

Innovation as a business model: One of the main objectives of companies is to maintain and expand business relationships with their customers. Value-added services and new business models are a fundamental tool to achieve this and represent new opportunities. IoT technologies allow offering proposals to old customers and attracting new ones, enriching their services, obtaining more information, improving efficiency, and differentiating from competitors [20].

Entrepreneurial spirit: The connected world is highly volatile and dynamic, and the task of entrepreneurs and managers is to shape it by constantly taking advantage of the changes that are presented. This includes being prepared to deal with new environments that are presented at high speed in much the same way as when a new company is launched. Thus, IoT makes recommendable business structures divided into small, independent, agile business units that continually explore their environments and learn to prevent and minimize risks, but at the same time from a global perspective.

The use of Open source tools and cheap devices: One possible approach is to use open source software tools and nonexpensive devices (such raspberry Pi) to deploy IoT solutions and integrate them with existing machines and systems in industries, in order to achieve full coverage of the organization's activity. It is necessary to adapt the elements that the industry already has to new technologies; this allows a reuse that not only economically favors companies, but also reduces the impact of discarding them.

\section{B. Blockchain Technology}

1) Theoretical overview: Blockchain is commonly acknowledged as the technology behind Bitcoin [37]. However, the possible applications of Blockchain are much more than merely facilitating digital currencies [38]. Blockchain allows a growing set of parties to keep a tamperproof, safe, and permanent transactions' ledger without a chief authority. Moreover, the transactions are not noted centrally, which is a vital aspect of this technology [39].

The technology of Blockchain unlocks various prospects to reform collaborative business processes, such as logistics and supply chain processes. These kinds of processes are usually performed by depending on reliable third-party providers, such as electronic data interchange escrows or hubs. The central design forms barriers to entry and deters process innovation. With the help of Blockchain, these processes can be performed in a peer-to-peer style without entrusting trust to central authorities nor needing mutual trust amid all groups of parties [40].

Prior works [41-43] show the viability of conducting collaborative business processes on a Blockchain platform. This is done by altering a collaborative process model into a smart contract serving like a template. After this, instancespecific smart contracts are produced from this template, which is done to observe or perform all instances of the process. This primary proof-of-concept design has bought out the requirement to enhance the usage of resource. Certainly, the Blockchain technology's cost is extremely subtle to the size of data registered on the ledger as well as the rate with which the smart contracts update the data [44,39]. Reducing the code size, rate of data writes, and data retained in the smart contracts is essential in making the Blockchain technology a feasible substitute for performing collaborative business processes.

2) Blockchain technology improvement opportunities for BPM: What kind of business process enhancement prospects does the Blockchain technology allow? To answer this, the value-driven BPM (VBPM) structure is used[45]. The VBPM structure (Fig. 7) categorizes the value, which the BPM can obtain for a business in seven groups. The "transparency" value is the main value in this framework, and states the capability for BPM to assist the shareholders of a business to monitor as well as comprehend their business processes in a better way.

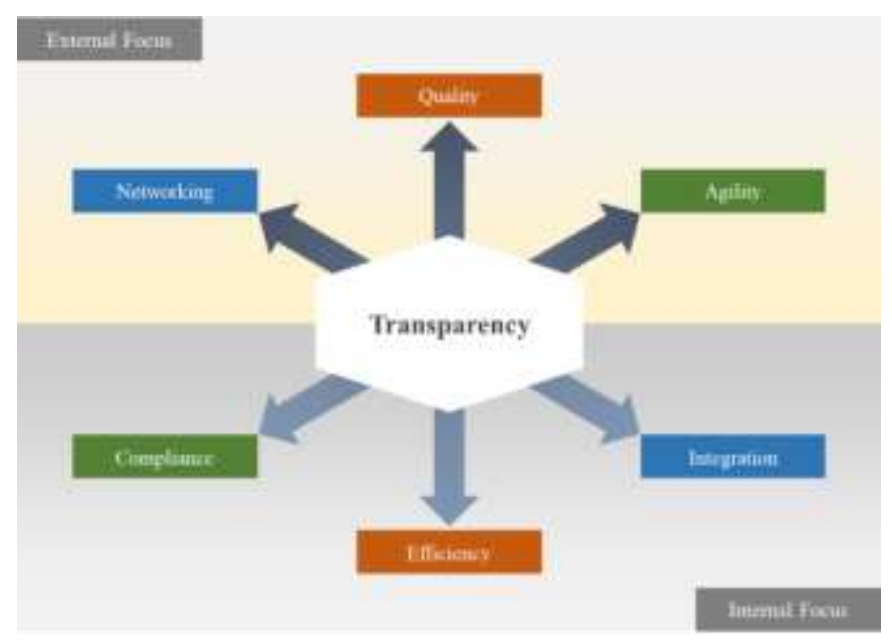

Fig. 7. Value-Driven BPM, Adapted from [45]. 
After the transparency is attained, BPM can bring value in three axes, with every axis equating to a pair of values. The efficiency-quality pair is the first axis, which shows a regularly recognized dichotomy amid efficiently satisfying the customer's needs (quality) and restructuring the processes to proficiently satisfy these needs (like, at minimum cost). The agility-compliance pair is the second axis, which takes the contrast amid being capable to quickly adjust to a varying business environment (flexibility) vs. protecting the processes to make sure that they obey the applicable standards and rules. The third axis (integration-networking pair) takes the double imperative for businesses to keep their inner systems combined to avert siloes and depict the systems to external business partners to make use of collaborative business prospects [46].

Efficiency vs. quality: Several cases are suggested for Blockchain emphasis on augmenting competence by decreasing costs and time. The business of banking has profoundly invested in start-ups based on Blockchain, which functions with forming new arrangements for clearing of fiscal products. In partnership with more than 40 banks, the Corda platform has been presented by R3. The platform can re-design the present settlement processes by allowing the registration of financial agreements once, thus, removing the repetition of data to save costs and time from redundant reconciliation processes. Similarly, the Australian Stock Exchange is keenly looking to substitute its established CHESS payment system with a distributed ledger based on Blockchain to decrease costs and settlement times [39].

Blockchain can also improve the quality of the consequence of business processes. When it comes to supply chains, tracking of materials and goods based on Blockchain could let a thorough tracking as well as avert fake products getting into the chain and avert replacement of good quality with inferior quality materials. One more instance is from the insurance sector, in which the eminence of the claim-handling course could be enhanced with the usage of the Blockchain technology. Blockchain would offer means to avert the usage of outdated or flawed data in the procedure.

Agility versus compliance: Agility is the ease and speed with which a business can retort to diverse modifications. The technology of Blockchain lets "smart contracts," which automatically perform particular actions as mentioned in its conditions. These kinds of constructs could advance the agility of a course by shifting run-time implementations to smart contracts, thus describing the procedure per contract instead of the entire system. For example, with the goal of attaining safer, tailored, and auditable pay-outs, the UK government wants to put their welfare payment procedure on Blockchain.

The Blockchain technology can also make the compliance simpler by letting the dispensing of private data safely. Financial businesses, which are frequently put through new compliance guidelines, could dispense the data on Blockchain and give admission to financial regulatory agencies. It would eliminate the requirement of recording by giving the work of collecting data to the regulatory activities. This principal solution can be put on the accounting data of companies and their auditors.
Integration vs. networking: Blockchain could also be made use of to allow an enhanced incorporation of the processes in a firm. Blockchain has also been suggested for linking software, which will allow an inner network, in which the information systems are internally connected for data sharing. Although this practice is not much endorsed, the Blockchain's networking feature is widespread [46].

The use of Blockchain as a registry of products is one of the most indorsed use. The notion is to record, register, and look into the transference of the possession of any product on distributed ledgers. Some of the benefits are being tamperproof, avoiding third-party central ledgers, security, and clean records [47]. Nevertheless, this kind of solution allows collaborative processes. A research is taking place in Sweden to list and record land titles on a Blockchain solution. This will allow multi-party partnership, in which government agencies, banks, sellers, and buyers can cooperate and look at the development of the procedure.

The ability to considerably alter an extensive spectrum of business processes lies with the Blockchain technology [47]. With Blockchain making its presence known, it will possibly be the job of process experts to make sure that the value formed originates from discovering the prospects that Blockchain can support in business processes in place of merely substituting present technologies with others.

3) Blockchain and BPM capabilities and challenges: When new technologies show up, which are still in the first stages and far from reaching completeness, there is always a lot of debate $[48,11]$. In this section, the challenges and the opportunities that come from the Blockchain technology regarding the six BPM core competence areas are discussed. Therse core competencies are presented in Fig. 4, namely culture, methods, strategic alignment, information technology, governance, and people.

Strategic alignment: The Blockchain technology needs a study on how systematic analyses of the strategic effects of its usage on particular processes could be directed. As an unruly technology, Blockchain puts queries if the traditional process following a strategy model could be flipped with new processes based on Blockchain, challenging whole businesses like accounting. For numerous businesses, a possible disintermediation that could be allowed by the usage of Blockchain-based systems may result in a threat, and not an opportunity [11].

Governance: The Blockchain technology alters governance for the reason that it allows coopetition as a novel management style for processes [49]. This needs research on the description of devoted roles that communicate with external and internal partners for putting Blockchain provision for processes. Moreover, policies that describe when and where the Blockchain technology could be made use of or should not be made use of are needed for backing processes. For example, cryptocurrencies take exchange rates that are extremely volatile to old-style currencies, gains, and losses of $10-50 \%$ in a day are not rare. This instability would likely reduce with a wider uptake. At this time, it is a barricade for numerous applications. 
Novel attack circumstances on Blockchain networks are hard to predict. As a result, strategies for making use of consortium, public-, or private-based Blockchains are needed. Lastly, smart contracts assure to ease self-governance of not merely processes, but of whole groups, like those presently role-modeled by the Decentralized Autonomous Organization. Making a decision on which applicants must have the discernibility of the parts of the transaction history also postures tests in trade-offs. On the one hand, advanced confidentiality and privacy will upsurge the receipt of the technology. On the other hand, more clearness in results in honesty, agreement checking, and optimization are founded on consistent data [50].

Methods: The Blockchain technology would need novel analysis approaches precisely with a focus on cost/benefit study and risk assessment and collaborative engineering approaches [44]. These need official reasoning competences regarding the precision and privacy protection of smart contracts. In addition, Blockchain would transmit attention from examining pain points back to looking for uncharted new prospects that may recover the certainty of the process reengineering ideas argued in the early 1990s.

Information technology: The Blockchain technology needs novel answers, software components, and integrated development environments to apply business processes with Blockchains. Moreover, novel challenges have come out with issues of privacy and security, such as how to avert private business data leak. Though the discernibility of encoded data on a Blockchain is limited, it is in the hands of the contestants in the process to make sure that these devices are made use of in accordance to their confidentiality necessities. The certainty of these necessities is presently examined in the fiscal sector. Lastly, the characteristic boundaries of Blockchains are to be deliberated comprising throughput, computational power, processing costs, and data storage. Instead of making use of a present Blockchain, a substitute can be used to simply accept equivalent design philosophies, such as virtual transaction history [47].

People: The acceptance of Blockchains and the scheme of smart contracts would need novel means of thinking regarding individuals as the emphasis moves from processes in a sphere of control to alliance amid groups. Furthermore, individuals should be keen to make business partnerships in the frame of present rules to assist acceptance. This suggests that investigation of the Blockchain technology acceptance is required. Lastly, with inferences for work associations, giving incentives to third parties to add to current processes becomes simple because of the sincerity of Blockchains.

Culture: Blockchains need research in the organizational aspects, which ease initial and fruitful adoption. The organizational culture can probably be one of the aspects because the Blockchain model defies classical organizational structures [49].

Current research in the field of integrating a Blockchain into BPM is just beginning. At the moment there are still gaps in research and a lack of experience in this area. For the productive use of the Blockchain technology, the abovementioned challenges will have to be mastered in the future. For example, there is currently a great need for specific requirements, conceptual models, procedures, case studies and technical solutions for successful integration into the system landscape of large companies and into the supplier network. A key aspect here is the integration of the Blockchain into the existing system landscapes.

Another practical example is connecting the Blockchain to the existing ERP systems; there are synergy effects for the improvement and efficient handling of the current process steps. When integrating both approaches, high care should be taken to ensure that the different concepts of the two systems harmonize with one another and are coordinated with one another. This is the only way to utilize the advantages and potential of both of them in an optimal way.

4) Key success factors: The biggest key success factor to turning Blockchain's potential into reality in BPM is the collaborative effort between organizations. Although business networks are a critical success factor for the adoption of the Blockchain technology, many other factors should also be considered [48]. One of these factors is the generation of an internal drive for the integration and application of Blockchain among enterprises, a factor that many organizations are struggling to achieve today [11]. Additional success factor that should be discussed is whether Blockchain capabilities contradiction with security challenges. Security is another reason why organizations might hesitate to adopt Blockchain, which is also reasonable. Ensuring process both secure and efficient is a challenge in itself, and it is a success factor for Blockchain generally. Although is not easy, however, it is not impossible as we have been a witness for many invocations in the usability of more secure solutions in the past years and the Blockchain will not be an exception. Beyond these factors, something is more fundamental: the need to make stakeholders within the organization be aware of the potential benefits of Blockchain and its positive effects. Finally, in order to apply Blockchain successfully, a common coordination among organizations must be fostered, which is vital in generating the positive network effects that make Blockchain very compelling [39].

\section{DISCUSSION}

BPM is one of the most pronounced topics when dealing with information technology applied to the business environment. This paradigm encompasses the concepts, methods, techniques and software tools that support the life cycle of an organization's business processes. BPM is considered a multidisciplinary approach since it presents connectors with highly related business and technological elements. Along with the technological connectors as a type of application integration technologies incorporated into BPM, many merging technologies such Blockchain and IoT have evolved to change the way BPM is operated and managed, constitute a means to lead organizations to be more agile and innovative. This article conducted a review on how BPM could be integrated with the Blockchain and IoT technologies. It highlights the challenges that face the integration process, 
describes and discuss the key success factors of the integration between the technologies under investigation.

Few reviews analyze the factors that influence the adoption of BPM technologies and emerging systems in the literature consulted. Reviews in this domain of knowledge focus on: assessing the characteristics of BPM technologies based on their proprietary or free nature [51, 52], analyzing their architecture and standards [53], and evaluating business process maturity models as evidence of its utilitarian nature and in relating BPM to software engineering in topics such as requirements engineering or the approach to software product lines.

In this section, a summary for our review on integrating Blockchain and IoT with BPM are listed:

- The first technology is the Internet-of-Things, which has the potential to fundamentally change the way people interact with their environment. IoT makes it possible to consider extensive real-time information in business processes. The result is processes that are more transparent and monitoring of business processes. The processes have to be adapted to the new possibilities of IoT to realize their full potential. The right people must use the right objects in the right situation at the right time. Therefore, the use of IoT requires flexible business processes that are capable of dynamically adapting to the changes that can occur through process execution or the properties of the smart objects. Numerous difficulties and challenges need to be investigated during the adoption of IoT for BPM, such as: extending and enhancing the current business process modeling and execution and service description languages, dealing with unreliable data, dealing with unreliable resources, and performing highly distributed processes.

- The second technology is the Blockchain which unlocks various prospects to reform collaborative business processes, such as logistics and supply chain processes. Moreover, Blockchain allows a growing set of parties to keep a tamperproof, safe, and permanent transactions' ledger without a chief authority. Moreover, the transactions are not noted centrally, which is a vital aspect of this technology. Some related challenges are as follow: Blockchain technology needs a study on how systematic analyses of the strategic effects of its usage on particular processes could be directed. Also consider the needs to a description of devoted roles that communicate with external and internal partners for putting Blockchain provision for processes. Finally, the policies that describe when and where the Blockchain technology could be made use of or should not be made use of are needed for backing processes and the needs for novel analysis approaches precisely with a focus on cost/benefit study and risk assessment and collaborative engineering approaches.

The research results should be interpreted taking into account some limitations, mainly with respect to the underlying research method. Specifically, the application of content analysis does not allow the study to be exempted from a certain subjectivity. In order to avoid or minimize it, the authors have carried out this analysis, with an additional volunteer researcher intervening to coordinate the solution to discrepancies or different interpretations when quantifying the information. Similarly, another limitation is the number of databases consulted and that only academic articles published in journals and peer-reviewed have been searched. Perhaps, if the search is extended to a greater number of repositories and other types of publications (books, book chapters, contributions to congresses, doctoral theses or even informative works), more works could be found that confirm the findings and results of different research aspects. On the contrary, they suppose a new perspective for the literature in the field. In any case, it was decided to include only academic articles published in journals to guarantee their quality.

\section{CONCLUSION}

The digital transformation is revolutionizing every industry. The change is occurring at an exponential pace. Enterprises embarking on this new world have unlimited possibilities. New ways to connect with customers, more innovation for the workforce, the chance to engage and retain the most talented people, and the use of data analytics are just some of the possibilities of digitization. The benefits are clear: customers benefit from a successful delivery of higher sales, and can differentiate themselves from the competition in the market. The objective of this work was to investigate two emerging technologies and explain how they can be applied to improve business process management by studying their opportunities, challenges, and key success factors.

As a future work, more investigations on the impact of Blockchain and IoT on BPM can applied through a qualitative and quantitative study on organizations of different sizes. Additional emerging technologies such as Cloud computing can be investigated also. Finally, reviewing more surveys that are recent and relevant could add a higher value to the overall work.

\section{REFERENCES}

[1] C. Chang, S. N. Srirama, and R. Buyya, Mobile Cloud business process management system for the internet of things: a survey, ACM Computing Surveys, vol. 49, no. 4, pp. 70, 2017.

[2] M. Rosemann, and J. vom Brocke, The six core elements of business process management, Handbook on business process management, In Handbook on business process management 1, Springer, pp. 105-122, 2015.

[3] J. vom Brocke, and J. Mendling, Frameworks for business process management: a taxonomy for business process management cases, Business Process Management Cases, Springer, , pp. 1-17, August, 2017.

[4] L. Da Xu, Enterprise systems: state-of-the-art and future trends, IEEE Transactions on Industrial Informatics, vol. 7, no. 4, pp. 630-640, 2011.

[5] M. Yang, and P. Gabrielsson, Entrepreneurial marketing of international high-tech business-to-business new ventures: A decision-making process perspective, Industrial Marketing Management, vol. 64, pp.147-160, July, 2017.

[6] K. Korpela, J. Hallikas, and T. Dahlberg, Digital supply chain transformation toward blockchain integration., In proceedings of the 50th Hawaii international conference on system sciences, Village, Hawaii, , January, 2017.

[7] J. F. Chang, Business process management systems: strategy and implementation:, CRC Press, , April, 2016. 
[8] N. J. A. S. Kratzke, A brief history of cloud application architectures, Applied Sciences, vol. 8, no. 8, pp. 1368, 2018.

[9] L. $\mathrm{Da} \mathrm{Xu}, \mathrm{W}$. He, and $\mathrm{S}$. Li, Internet of things in industries: A survey, IEEE Transactions on industrial informatics, vol. 10, no. 4, pp. 22332243, 2014.

[10] W. M. Van der Aalst, Business process management: a comprehensive survey, ISRN Software Engineering, vol. 2013, , 2013.

[11] W. Viriyasitavat, L. Da Xu, Z. Bi, and A. Sapsomboon, Blockchainbased business process management (BPM) framework for service composition in industry 4.0, Journal of Intelligent Manufacturing, vol. 2018, No.1, pp. 1-12, May, 2018.

[12] M. Klun, and P. Trkman, Business process management-at the crossroads, Business Process Management Journal, vol. 24, no. 3, pp. 786-813, 2018.

[13] C. Chang, S. N. Srirama, and R. Buyya, Mobile Cloud Business Process Management System for the Internet of Things: A Survey, ACM Computing Surveys, vol. 49, no. 4, pp. 1-42, 2016.

[14] J. Luftman, K. Lyytinen, and T. ben Zvi, Enhancing the measurement of information technology (IT) business alignment and its influence on company performance, Journal of Information Technology, vol. 32, no. 1, pp. 26-46, 2017.

[15] W. van der Aalst, A. Ter Hofstede, and M. Weske, Business Process Management:, International Conference, BPM 2003, Proceedings: Springer Science \& Business Media, , Eindhoven, The Netherlands, June 26-27, 2003.

[16] W. Viriyasitavat, L. Da Xu, Z. Bi, and V. Pungpapong, Blockchain and Internet of Things for Modern Business Process in Digital Economy--the State of the Art, IEEE Transactions on Computational Social Systems, vol. 6, No. 6, pp. 1420-1432, 2019.

[17] H. Pervez, and I. U. Haq, Blockchain and IoT Based Disruption in Logistics., In 2019 2nd International Conference on Communication, Computing and Digital systems (C-CODE), Pakistan, Islamabad, pp. 276-281, March, 2019.

[18] J. Gubbi, R. Buyya, S. Marusic, and M. Palaniswami, Internet of Things (IoT): A vision, architectural elements, and future directions, Future generation computer systems, vol. 29, no. 7, pp. 1645-1660, 2013.

[19] L. Atzori, A. Iera, and G. Morabito, The internet of things: A survey, Computer networks, vol. 54, no. 15, pp. 2787-2805, 2010.

[20] S. Meyer, A. Ruppen, and C. Magerkurth, Internet of things-aware process modeling: integrating IoT devices as business process resources., In International conference on advanced information systems engineering, pp. 84-98, Springer, Berlin, Heidelberg, 2013.

[21] Y.-T. Chen, and M.-S. Wang, A study of extending BPMN to integrate IoT applications., In 2017 International Conference on Applied System Innovation (ICASI), Sapporo, Japan, pp. 1797-1800, May 13-17, 2017.

[22] C. Janiesch, A. Koschmider, M. Mecella, B. Weber, A. Burattin, C. Di Ciccio, A. Gal, U. Kannengiesser, F. Mannhardt, and J. Mendling, The internet-of-things meets business process management: mutual benefits and challenges, arXiv preprint, arXiv:1709.03628, , 2017.

[23] E. d. S. Zancul, S. M. Takey, A. P. B. Barquet, L. H. Kuwabara, P. A. Cauchick Miguel, and $\mathrm{H}$. Rozenfeld, Business process support for IoT based product-service systems (PSS), Business Process Management Journal, vol. 22, no. 2, pp. 305-323, 2016.

[24] S. Haller, and C. Magerkurth, The real-time enterprise: Iot-enabled business processes., In IETF IAB Workshop on Interconnecting Smart Objects with the Internet, Prague, pp. 1-3, 25th March 2011.

[25] H.-H. Chiu, and M.-S. Wang, A study of iot-aware business process modeling, International Journal of Modeling and Optimization, vol. 3, no. 3, pp. 238, 2013.

[26] M. Del Giudice, Discovering the Internet of Things (IoT): technology and business process management, inside and outside the innovative firms, Business Process Management Journal, vol. 22, no. 2, 2016.

[27] M. Schief, C. Kuhn, P. Rösch, and T. Stoitsev, Enabling Business Process Integration of IoT-events to the Benefit of Sustainable Logistics., In the International Conference on Logistics, Informatics and Service Science (LISS 2015), Beijing, China, pp. 40-49, February, 2011.
[28] S. Appel, P. Kleber, S. Frischbier, T. Freudenreich, and A. Buchmann, Modeling and execution of event stream processing in business processes, Information Systems, vol. 46, pp. 140-156, 2014.

[29] A.-M. Barthe-Delanoë, S. Truptil, F. Bénaben, and H. Pingaud, Eventdriven agility of interoperability during the Run-time of collaborative processes, Decision Support Systems, vol. 59, pp. 171-179, 2014.

[30] R. G. Lee, and B. G. Dale, Business process management: a review and evaluation, Business process management journal, vol. 4, no. 3, pp. 214$225,1998$.

[31] G. Goldkuhl, and P. J. Ågerfalk, Action within information systems: outline of a requirements engineering method., In the Fourth International Workshop on Requirements Engineering: Foundation for Software Quality, Pisa, Italy, pp. 133-153, June 8-9 1998.

[32] Y. Cheng, S. Zhao, B. Cheng, S. Hou, Y. Shi, and J. Chen, Modeling and Optimization for Collaborative Business Process Towards IoT Applications, Mobile Information Systems, vol. 2018, , 2018.

[33] M. Papert, A. Pflaum, and A. Leischnig, A business process perspective on IoT implementation: findings from a comparative case analysis, International Conference on Information Systems (ICIS), Seoul, , December 10th- 13th, 2017.

[34] S. Haller, S. Karnouskos, and C. Schroth, The internet of things in an enterprise context., In Future Internet Symposium, Springer, Berlin, Heidelberg, pp. 14-28, 29 September, 2008.

[35] S. Schönig, L. Ackermann, S. Jablonski, and A. Ermer, An integrated architecture for IoT-aware business process execution, In Enterprise, Business-Process and Information Systems Modeling, , pp. 19-34, Springer, Cham, 2018.

[36] H.-G. Hong, Business Process Support Based on IoT Technology, Journal of Convergence for Information Technology, vol. 7, no. 1, pp. 75-79, 2017.

[37] Eyal, A. E. Gencer, E. G. Sirer, and R. Van Renesse, Bitcoin-NG: A Scalable Blockchain Protocol., In 13th \{USENIX\} symposium on networked systems design and implementation (\{NSDI\} 16), Santa Clara, CA, USA, pp. 45-59, March 16-18, 2016.

[38] D. Tapscott, and A. Tapscott, Blockchain Revolution: How the technology behind Bitcoin is changing money, business, and the world: aPenguin, The Quality Management Journal, Vol. 25, no. 1, pp.64-65, 2016.

[39] Weber, X. Xu, R. Riveret, G. Governatori, A. Ponomarev, and J. Mendling, Untrusted business process monitoring and execution using blockchain., In International Conference on Business Process Management, Springer, Cham, pp. 329-347, 2016, September.

[40] R. Hull, Blockchain: Distributed Event-based Processing in a DataCentric World., In Proceedings of the 11th ACM International Conference on Distributed and Event-based Systems, Barcelona, Spain, pp. 2-4, June, 2017.

[41] L. García-Bañuelos, A. Ponomarev, M. Dumas, and I. Weber, Optimized execution of business processes on blockchain., In The International Conference on Business Process Management, Barcelona, Spain, pp. 130-146, September 10-11, 2017.

[42] C. Prybila, S. Schulte, C. Hochreiner, and I. Weber, Runtime Verification for Business Processes Utilizing the Bitcoin Blockchain, arXiv preprint, rXiv:1706.04404, 2017.

[43] D. Verma, N. Desai, A. Preece, and I. Taylor, A blockchain based architecture for asset management in coalition operations., In Ground/Air Multisensor Interoperability, Integration, and Networking for Persistent ISR VIII, International Society for Optics and Photonics, vol. 10190, pp. 101900Y-101900Y-9, May, 2017.

[44] J. Mendling, I. Weber, W. V. D. Aalst, J. V. Brocke, C. Cabanillas, F. Daniel, S. Debois, C. D. Ciccio, M. Dumas, and S. Dustdar, Blockchains for business process management-challenges and opportunities, ACM Transactions on Management Information Systems (TMIS), vol. 9, no. 1, pp. 4, 2018.

[45] F. Milani, L. García-Bañuelos, and M. Dumas, Blockchain and business process improvement, BPTrends newsletter, , October, 2016.

[46] López - Pintado, L. García - Bañuelos, M. Dumas, I. Weber, and A. Ponomarev, CATERPILLAR: A business process execution engine on the Ethereum blockchain, Software: Practice and Experience, vol. 49, no. 7, pp. 1162-1193, 2019. 
[47] B. Tran, Q. Lu, and I. Weber, Lorikeet: A Model-Driven Engineering Tool for Blockchain-Based Business Process Execution and Asset Management., In BPM (Dissertation/Demos/Industry), , pp. 56-60, 2018.

[48] S. Saberi, M. Kouhizadeh, J. Sarkis, and L. Shen, Blockchain technology and its relationships to sustainable supply chain management, International Journal of Production Research, vol. 57, no. 7, pp. 2117-2135, 2019.

[49] J. Mendling, I. Weber, W. van der Aalst, J. v. Brocke, C. Cabanillas, F. Daniel, S. Debois, C. Di Ciccio, M. Dumas, and S. Dustdar, Blockchains for Business Process Management-Challenges and Opportunities, arXiv preprint, arXiv:1704.03610, , 2017.

[50] Norta, Establishing Distributed Governance Infrastructures for Enacting Cross-Organization Collaborations., In International Conference on
Service-Oriented Computing, Springer, Berlin, Heidelberg, pp. 24-35, November, 2015.

[51] Sousa, Marco, et al. "Evaluation of BPM tools open source/freeware." 2018 13th Iberian Conference on Information Systems and Technologies (CISTI). IEEE, 2018.

[52] Pourmirza, S., Peters, S., Dijkman, R., \& Grefen, P. (2017). A systematic literature review on the architecture of business process management systems. Information Systems, 66, 43-58.

[53] Correia, E., Carvalho, H., Azevedo, S. G., \& Govindan, K. (2017). Maturity models in supply chain sustainability: A systematic literature review. Sustainability, 9(1), 64. 